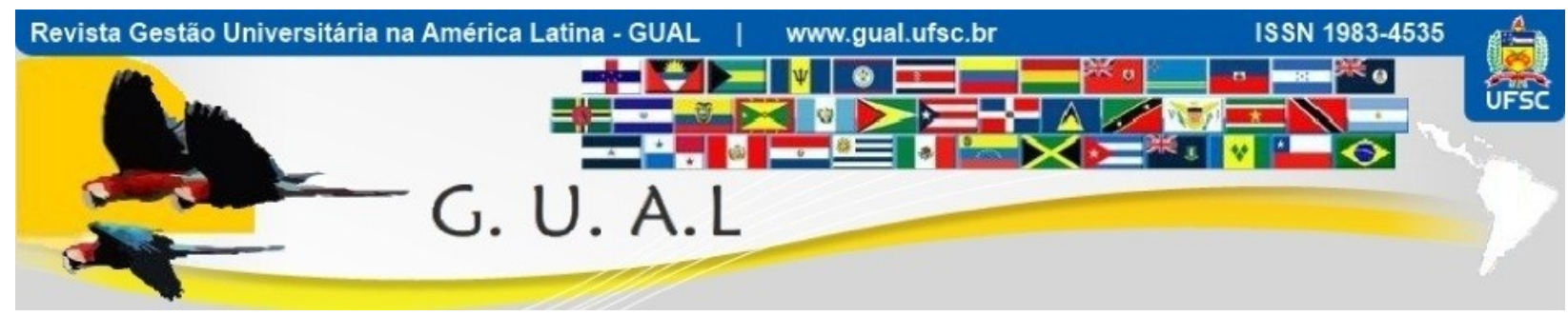

DOI: http://dx.doi.org/10.5007/1983-4535.2012v5n4p266

\title{
IMPORTANCIA DE LA RESPONSABILIDAD SOCIAL UNIVERSITARIA COMO DESARROLLO SOCIAL DE LA CIUDAD DE CORONEL OVIEDO
}

THE IMPORTANCE OF SOCIAL RESPONSIBILITY IN THE UNIVERSITY AS A PART OF SOCIAL DEVELOPMENT IN THE CITY OF CORONEL OVIEDO

\author{
Hugo Alfredo Recalde, Mestre \\ Universidad Nacional de Caaguazú - UNCA \\ vicerector.unca@gmail.com \\ Willian Cantero Lusardi, Mestre \\ Universidad Nacional de Caaguazú - UNCA \\ williancantero@gmail.com \\ Juan Jara, Mestre \\ Universidad Nacional de Caaguazú - UNCA \\ jaraju01@gmail.com
}

Recebido em 16/novembro/2012

Aprovado em 07/dezembro/2012

Sistema de Avaliação: Double Blind Review

Esta obra está sob uma Licença Creative Commons Atribuição-Uso. 


\title{
RESUMEN
}

Dentro del marco de la responsabilidad social universitaria la UNC@, La Dirección de Investigación Científica realizo un "Estudio de Prevalencia de Parásitos Intestinales en Escolares de la Cuidad de Coronel Oviedo, se analizaron 638 muestras. Siendo los resultados: el 24\% de los niños estaban parasitados, 45\% de Áscaris lumbricoides, 35\% de Giardia lamblia, $16 \%$ de Hymenolepis, $4 \%$ de Ansylotoma duodenale, de ello nace el proyecto un cambio en el barrio 1 de Marzo que consistió en talleres de capacitación en las áreas de las Ciencias Medicas, Ciencias Tecnológicas y Ciencias Económicas, dentro del campo de acción del conocimiento de cada Facultad. El trabajo se dividió en 2 etapas, la concienciación de la población estudiantil a través de charlas educativas impartidas por los becarios de la FCM, la segunda etapa en la realización del control antropométrico, administración de la dosis de antiparasitario y control de liendres de piojos con la entrega de los insumos para el tratamiento de los piojos. En esta actividad se invirtieron 56 horas distribuidas en 38 actividades, con una participación de aproximadamente 1.310 personas. De esta lógica de intervención se trabajo el componente de la transformación cultural de las personas al insertar en ellos las practicas de prevención y promoción de la salud, de esta manera la Universidad Nacional de Caaguazú cumple con la función integradora de la RSU.

Palabras-clave: Responsabilidad social universitaria. Facultad de Ciencias Médicas. Desarrollo social. Transformación cultural.

\begin{abstract}
Within the framework of the University's Social Responsibility initiative atUniversidad Nacional de Caaguazú(UNC), the Scientific Research Directorate program conducted a "Study of Prevalence of Intestinal Parasites in school children of the City of Coronel Oviedo".Of the 638 samples that were analyzed,24\% of children were infected with parasites; 45\% having Ascarislumbricoides, 35\% having Giardia lamblia, 16\% having Hymenolepis, and 4\% infected with Ansylotomaduodenale. Resulting from this study, workshops in the areas of Medical Sciences, Technology and Economics were started in the district of Primero de Marzo. The work was divided into two stages; the first was raising the awareness of the student population through educational lectures by scholars of the FCM, the second stage in the implementation of the project was administering dosage of the anti-parasitic medicine and controlling lice, nits, and delivery of supplies for the treatment of each. Approximately 1,310 people participated in this activity; investing 56 hours distributed between 38 activities. An important component of this project is the cultural transformation of the communityinvolved to change their daily practices in prevention of illness and promotion of a healthy lifestyle. In this manner, Universidad Nacional de Caaguazú (UNC) complies with theUniversity's Social Responsibility initiative.
\end{abstract}

Keywords: University's Social Responsibility initiative. Medical Sciences University. Social Development. Cultural Transformation. 


\section{LA RESPONSABILIDAD SOCIAL Y SU VINCULACION CON LA UNIVERSIDAD}

Tal como lo expresa François Vallaeys en su ensayo, de todo lo que se ha venido hablando y planteando en los últimos tiempos sobre la "responsabilidad social", la misma puede ser entendida como una dimensión ética que toda organización o institución debería tener como visión y que debería promover en su actividad diaria. Pero estas acciones diarias no deberían confundirse o asumirse, como estrategia de mercado o imagen institucional.

Para analizar el tema dentro de lo que se conoce hoy como Responsabilidad Social Universitaria(RSU), lo primero importante dentro del debate es visualizar y entender el concepto que se maneja de la Responsabilidad Social Empresarial(RSE), difundido mayormente en la actualidad y que puede ayudarnos a ilustrar los enfoques en las modernas organizaciones sobre la idea de "Responsabilidad Social".

La Responsabilidad Social Empresarial bien entendida y asumida supone un compromiso personal, empresarial y social, que promueve la calidad y competitividad apegada a la ley, y que va más allá promoviendo la práctica de valores éticos, con un reconocimiento de su implicancia social en las decisiones que asuma y que supone relaciones de mutuo beneficio entre todos los actores.

En un primer momento se afirma hoy que la RSE va más allá del altruismo. No quiere ser filantropía pura, es decir, lo que se conoce normalmente como donación caritativa y que no tiene relación alguna con la actividad empresarial, tampoco filantropía interesada, lo que significaría, la donación caritativa del producto de la empresa para promocionarlo o abrir nuevos mercados, los cuales no son sostenibles en el tiempo, pues no están relacionadas con los objetivos de la organización, y no están enmarcadas dentro de una visión integral e integradora de la sociedad y la organización dentro de la misma.

El libro verde de la Unión Europea la define cómo: concepto por el cual las empresas deciden contribuir voluntariamente a mejorar la sociedad y a preservar el medio ambiente. A través suyo, las empresas se conciencian del impacto de su acción sobre todos y expresan su compromiso de contribuir al desarrollo económico, a la vez que a la mejora de la calidad de vida de los trabajadores y sus familias, de la comunidad local donde actúan y de la sociedad en su conjunto. Otra definición expresa, la RSE la entenderemos como la acción conjunta de toda la empresa concienciada(trabajadores, directivos y dueños) del papel que tiene ésta como unidad de negocio que crea valor y que pervive en un espacio del que se lucra, y tiene que ver, en el ámbito social, con la ayuda a los más desfavorecidos y de respeto a los consumidores, ambiental, en la 
sostenibilidad y responsabilidad con el medio ambiente y, en lo económico, con las practicas leales, transparentes en el manejo de sus finanzas y de inversiones socialmente responsables.

Según Alejandra Ospina, los ámbitos de la RSE cubren 4 aspectos:

1. Aspecto laboral: cumplimiento de las normas de trabajo, respeto al trabajador en todos los niveles jerárquicos y puesta en marcha de códigos de conducta y principios de la Organización Internacional del Trabajo (OIT).

2. Aspecto económico: elaboración de cuentas transparentes y públicas, e inversiones socialmente responsables (utilizando criterios éticos y de exclusión, a la hora de invertir).

3. Aspecto ambiental: respetando el medio ambiente y usando sellos de calidad ecológica, o alusión directa al consumidor de cómo se obtuvo el producto, o se prestó el servicio.

4. Aspecto social: invertir un porcentaje de las ganancias en proyectos que ayuden a mejorar el nivel de vida de personas sin recursos. O tener proyectos de vinculación a colectivos desfavorecidos.

Dentro del alcance del concepto de RSE se plantean varios ejes transversales que fortalecen la idea de aplicabilidad, como ser:

a. Es una dimensión voluntaria que sobrepasa los establecimientos legales.

b. Se asocia con la idea del desarrollo sostenible, ya que sugiere proyectar acciones que integran el crecimiento económico, la equidad social y la prevención del medio ambiente.

c. Responde a una cobertura que contempla las realidades internas de la empresa y los espacios contextuales donde actúa. Grupos de Interés.

d. Afecta a la propia gestión de la organización.

Michael E. Porter y Mark R. Kramer, en su artículo "Estrategia y Sociedad” plantean que, los gobiernos activistas y medios de comunicación se han vuelto adeptos a perseguir rendición de cuentas de parte de las empresas por las consecuencias sociales de su actividad. Una multitud de organizaciones confeccionan rankings de empresas de acuerdo a sus desempeños en responsabilidad social corporativa, que concitan una considerable atención. Como resultado, la responsabilidad social corporativa ha emergido como una prioridad ineludible para los líderes de negocios en todos los países.

Las empresas ya han hecho y siguen haciendo mucho por mejorar las consecuencias sociales y medioambientales de sus actividades, pero estos esfuerzos, por un par de razones, están lejos de ser todo lo productivos que podrían. 


\section{IMPORTANCIA DE LA RESPONSABILIDAD SOCIAL UNIVERSITARIA COMO DESARROLLO SOCIAL DE LA CIUDAD DE CORONEL OVIEDO \\ DOI: http://dx.doi.org/10.5007/1983-4535.2012v5n4p266}

Primero, confrontan a las empresas con la sociedad, cuando obviamente ambos son interdependientes. Segundo, presionan a las empresas a pensar de manera genérica en responsabilidad social corporativa, en lugar de que lo hagan en la forma más apropiada para la estrategia particular de cada una, lo cierto es que los enfoques predominantes de Responsabilidad Social Corporativa están tan fragmentados y tan desconectados de los negocios y de la estrategia que oscurecen muchas de las mejores oportunidades que tienen las empresas para beneficiar a la sociedad.

Porter y Kramer también afirman que la mayor atención corporativa a la Responsabilidad Social Corporativa no ha sido totalmente voluntaria. Muchas empresas se dieron por enteradas sólo después de su sorpresa ante la respuesta pública por asuntos que anteriormente no habían considerado como parte de sus responsabilidades empresariales. Nike, por ejemplo, enfrentó un amplio boicot de los consumidores después que de The New York Times y otros medios reportaron prácticas laborales abusivas de algunos de sus proveedores indonesios a principios de los 90. La decisión de Shell Oil de hundir la plataforma petrolera en desuso Brent Spar, en el Mar del Norte, generó protestas de Greenpace en 1995 así como titulares noticiosos internacionales. Las empresas farmacéuticas descubrieron que la comunidad esperaba que tuvieran una respuesta frente a la pandemia de SIDA en Africa, aunque ello sucediera muy lejos de sus principales mercados y líneas de producto. Se responsabiliza actualmente a las empresas de comidas rápida y de alimentos preempacados por la obesidad y mala nutrición.

De todo esto lo que puede colegir es de que la sociedad espera que las decisiones empresariales en los negocios sea tomada en relación directa del impacto que pueda tener sobre los riesgos éticos, sociales y ambientales de sus decisiones.

Luego de este recorrido sobre la idea de Responsabilidad Social Empresarial, conforme a Vallaeys, se pueden destacar algunas características significativas y de suma utilidad para la Responsabilidad Social Universitaria:

- La Responsabilidad Social se desarrolla cuando una organización toma conciencia de sí misma, de su entorno, y de su papel en su entorno, de una manera global e integral y, a la vez, contagiarse en todas las partes de la organización.

- Esta toma de conciencia está ligada a preocupaciones tanto éticas como interesadas, de hacer las cosas bien para que todos los beneficiarios internos y externos de los servicios de la organización estén bien.

- En base a esta articulación, se definen ciertos principios y valores como parámetros de acción buena para la organización. Se incluyen estos en la estrategia global y el 
funcionamiento rutinario de la organización, para que se impregnen todos los ámbitos de las acciones de la organización y sus consecuencias económicas, sociales, laborales y ambientales.

- La ética practicada debe ser:

Una ética servicial y de responsabilidad por las consecuencias de las acciones emprendidas

Una ética del dialogo y consenso entre todos los participantes y afectados

Una ética de la complejidad, que necesita una visión holista y global de todos los entretegidos (complexus) de los cuales la organización participa

Una ética regulativa para la autoorganización, donde se pueda corregir permanentemente los errores y asegurar la sostenibilidad y el equilibrio de la organización.

Luego de analizar este marco general, se puede plantear un pensamiento sobre la Responsabilidad Social Universitaria. En este nuevo enfoque social, a la universidad se le está demandando fortalecer su rol social, desarrollar una cierta injerencia política orientada al cambio estructural y el de instituir un pensamiento complejo, interdisciplinario, para promover soluciones integrales.

Dentro de este complejo planteamiento global de una sociedad con mejores indicadores de desarrollo, con actores que generen y creen valor sus acciones de tal manera a mejorar la calidad de vida de la sociedad en su conjunto, para la Universidad es un reto, el fomentar el vinculo con la realidad social impregnado en sus procesos educativos, el de incorporar la solidaridad como experiencia de aprendizaje y formación profesional y, promover la incursión transversal de la Responsabilidad Social a nivel de las instancias de la organización académica, los diversos programas y en las tradiciones funcionales de la docencia y la investigación.

La incorporación de la Responsabilidad Social a la Universidad supone el manejo transparente y eficiente de los recursos que la sociedad le confía, la formación de profesionales competentes y socialmente responsables y el aporte reflexivo, propositivo y practico.

Al igual que la empresa que ha debido superar el enfoque filantrópico de la inversión social (como gasto extra) para entenderse a sí misma bajo el nuevo paradigma de la Responsabilidad Social, la Universidad debe tratar de superar el enfoque de la "proyección social y extensión universitaria" como "apéndices" bien intencionados a su función central de formación estudiantil y producción de conocimientos, para poder asumir la verdadera exigencia de la Responsabilidad Social Universitaria. 
El punto de partida es una reflexión de la institución académica sobre sí misma en su entorno social, un análisis de su responsabilidad y sobre todo de su parte de culpabilidad de los problemas crónicos de la sociedad, dejando de pensarse como una burbuja de paz y racionalidad en medio de la tormenta en que se debate el "Titanic planetario", como llama Edgar Morin a nuestro barco Tierra, esa lujosa nave tecno - científica pero sin rumbo. La relación entre la crisis del saber tecno - científico hiperespecializado, pero fragmentado $\mathrm{y}$, su ceguera crónica concerniente a los efectos globales que engendra por un lado, y la crisis social y ecológica mundial por otro lado, tiene que ser el punto de partida para una reforma universitaria de responsabilización social que no sea meramente cosmética, sino una profunda reflexión sobre el significado social de la producción de conocimiento y la formación profesional de líderes en la era de la ciencia.

La experiencia chilena, "Universidad construye país", define que la Responsabilidad Social Universitaria exige, desde una visión holística, articular las diversas partes de la institución en un proyecto de promoción social de principios éticos y de desarrollo social equitativo y sostenible, para la producción y transmisión de saberes responsables y la formación de profesionales ciudadanos igualmente responsables.

Se puede visualizar en el figura siguiente, conforme al ensayo de Vallaeys, el carácter global y central de la reforma universitaria deseada.

Históricamente la Universidad en nuestro país como en la mayoría de los países latinoamericanos, ha contribuido en la formación académica de profesionales, con lo cual se ha ido respondiendo mínimamente a la demanda del mercado laboral, en su mayoría de ellos con alto conocimiento teórico, poco adiestradas en las soluciones prácticas que requieren las organizaciones y con casi nada de capacidades investigativas, por ende alejadas de la realidad del entorno, es decir, las instituciones de educación superior, docentes y alumnos que se convierten en una seudo elite social con barreras dogmaticas hacia la población más vulnerable, que no han permitido que la Universidad responda a los desafíos de la promoción del desarrollo.

Al decir de Jean Houssaye, estamos asistiendo a un derrumbe de lo que ha sostenido la escuela (Universidad). Progreso, cultura y moral ya no tienen lugar como tales, en el sentido de que ya no tienen consenso. Es el "progreso" el primero en ser discutido como referencia. Estamos en una sociedad tecnicista con gran desencanto, por lo cual es urgente elaborar nuevos valores para este nuevo mundo, por lo que la Universidad está llamada a ser actor fundamental en la construcción de soluciones para los nuevos desafíos sociales-globales. 


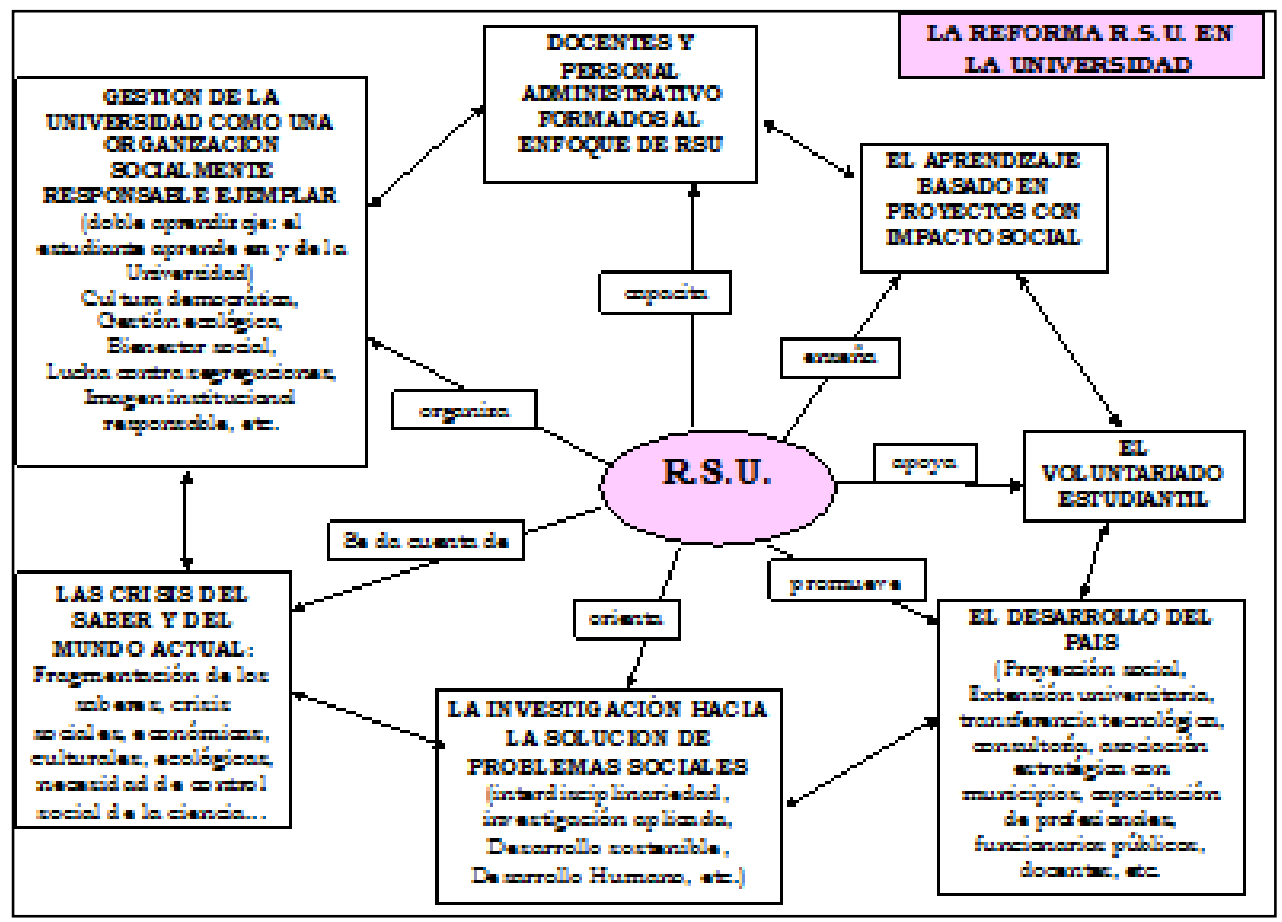

La legitimidad y la identidad de la universidad, en el proceso del tiempo se ha construido a partir de su vinculación con la adquisición, producción e innovación del conocimiento, por lo que resulta hasta imperativo en la actualidad que la misma esté llamada a dinamizar el desarrollo. La UNC@ para ser protagonista del desarrollo local debe fortalecer los procesos de adecuación académica-administrativa, formulando políticas que ayuden a establecer un relacionamiento Universidad-Sociedad, desde un enfoque Eco-socioconstructivista.

La Universidad Nacional de Caaguazú, interpretando los conceptos de Responsabilidad Social Universitaria en la actualidad y siendo protagonista, busca responder a los desafíos de la promoción del desarrollo, siendo "La Universidad Respuesta", con Facultades incluyentes que produzcan profesionales competentes y respondan a las demandas científicas, profesionales y educativas para un desarrollo local más justo y sostenible. En este marco, la UNC@ está trabajando en una Matriz de Calidad Universitaria donde los grandes desafíos son la GeneraciónProducción, Gestión-Administración y Transferencia-Aplicación del Conocimiento en la búsqueda del bienestar humano, para lo cual ha definido un cuarto "Pilar", DESARROLLO, a más de los que tradicionalmente se conocen, Academia, Investigación y Extensión. El Desarrollo entendido básicamente en tres aspectos; Cultural, Científico y Tecnológico, con la premisa del Desarrollo basado en el conocimiento y los procesos investigativos e innovación, para lo cual se deberán proyectar Centros especializados tales como: Laboratorios asociados, unidades de seguimiento de ex alumnos, oficinas de derechos intelectuales, unidades de gestión financiera, 
parques tecnológicos, incubadoras de emprendimientos, oficinas de derechos intelectuales, todos estos con un alto componente de relacionamiento Universidad-Empresa.

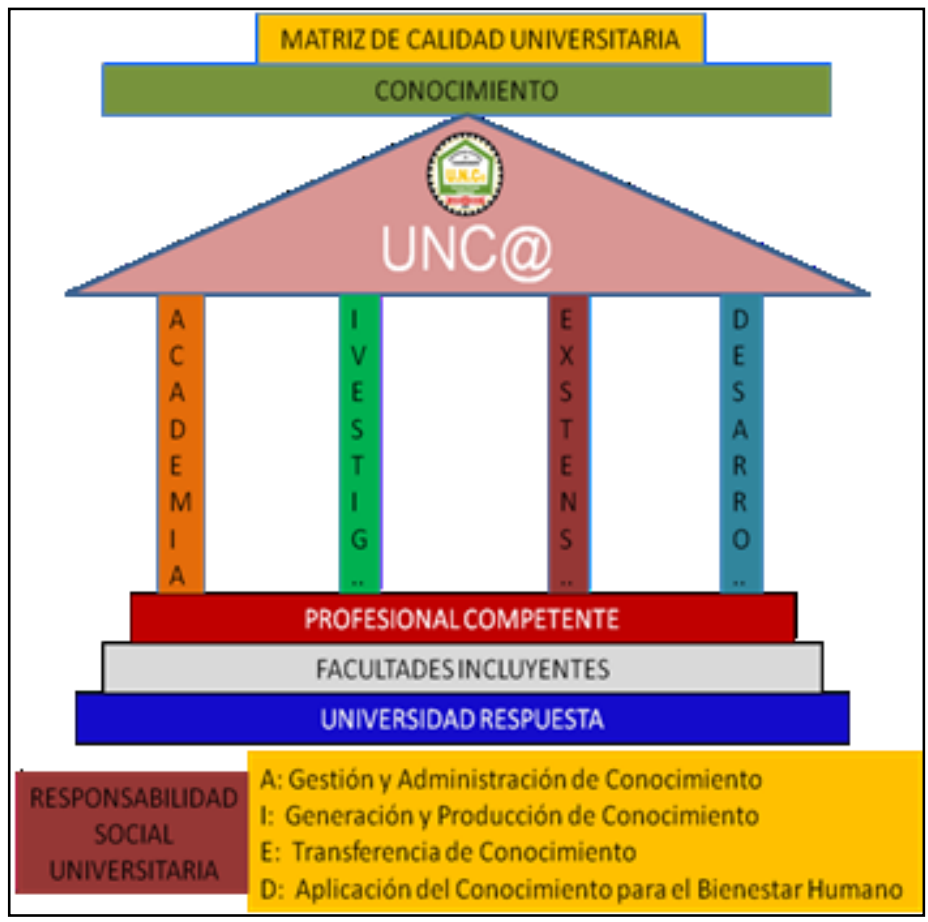

Por la importancia de su quehacer, la UNC@ tiene que desempeñar un papel central en la vida de la localidad y el país, de cara a afrontar problemas y condiciones globales.

De toda la teoría arriba expuesta nace la necesidad de poner en practica la responsabilidad social de la UNC@, y ello se concreta en Coronel Oviedo, que es una ciudad de Paraguay, capital del Departamento del Caaguazú, cuenta aproximadamente con 115.269 habitantes, según el censo 2002, actualmente conformada por más de 73 compañías, sus habitantes se dedican a la Industria, Comercio y servicios.

La Universidad Nacional del Caaguazú creada por ley 3198/2007 se halla ubicada en la ciudad de Coronel Oviedo con siete facultades y diez y ocho carreras, que entre sus objetivos principales se hallan consagrados la academia, la investigación, la extensión y el desarrollo, dentro de este proceso de desarrollo comunitario nace la extensión universitaria como respuesta a las necesidades de la comunidad y vinculo principal de la institución con el medio social. El rectorado cuenta con una dirección general de extensión universitaria y cada facultad con una dirección de extensión, quienes son las que realizan los trabajos operativos de los proyectos en la relación Universidad Sociedad. 
Dentro del marco de la responsabilidad social universitaria la UNC@, La Dirección General de Investigación Científica y el laboratorio de investigación en microbiología y parasitología Dr. "Kaneo Shibata" realizaron un "Estudio de Prevalencia de Parásitos Intestinales en Escolares de la Cuidad de Coronel Oviedo, en tres escuelas referenciales de la franja de pobreza de la cuidad, se tomo una muestra de heces por los alumnos de la Facultad de Ciencias Medicas para la búsqueda de parásitos intestinales, se analizaron 638 muestras. Los resultados encontrados fueron los siguientes: el $24 \%$ de los niños que ingresaron al estudio estaban parasitados, 45\% de Áscaris lumbricoides, 35\% de Giardia lamblia, 16\% de Hymenolepis, 4\% de Ansylotoma duodenale, agrupados por edades 3-5 años 11 parásitos, 6-9 años 96 parásitos, mayores a 948 parásitos. Siendo el índice más alto en la población de la escuela del Barrio 1ro. De Marzo. Este trabajo fue tomado como base para la preparación de un proyecto cuyo resultado directo fuera la minimización de la presencia de parásitos intestinales en la población educativa.

En relación a lo antes explicado nace el proyecto un cambio en el barrio 1 de Marzo que consistió en talleres de capacitación en las áreas de las Ciencias Medicas, Ciencias Tecnológicas y Ciencias Económicas, dentro del campo de actuación de cada Facultad; siendo los alumnos becados los principales protagonistas de la transferencia de conocimientos, supervisados por los docentes tutores, para con la comunidad y los principales referentes sociales del mismos: padres de familia, Directores de la escuela primaria, docentes y vecinos de la comunidad. Las jornadas estarán organizadas en grupos de trabajo en función de las actividades previstas para el logro de los objetivos.

La realización del trabajo se dividió en 2 etapas, la primera, la concienciación de la población estudiantil de la escuela $1^{\circ}$ de marzo a través de charlas educativas impartidas por los becarios de la FCM, estas charlas fueron dirigidas a mejorar los hábitos higiénicos que ayudan a evitar la parasitosis, tanto interna como externa, la segunda etapa en la realización del control antropométrico, administración de la dosis de antiparasitario y control de liendres de piojos con la entrega de los insumos para el tratamiento de los piojos.

El objetivo principal es fortalecer las capacidades del alumno en la gestión de la comunidad en las áreas de Facultad de Ciencias Medicas, Facultad de Ciencias Económicas, Facultad de Ciencias Tecnológicas con acciones coordinadas de extensión universitaria como responsabilidad social de la universidad, de igual manera el mejoramiento de las capacidades de la comunidad en las áreas de salud y socioeconómico, cultural y educación.

La lógica de la intervención fue la siguiente: 
- Concienciar sobre la importancia de los hábitos higiénicos para evitar la parasitosis, mediante charlas educativas.

- Fortalecer mediante talleres y capacitaciones en las áreas de educación y promoción de la prevención en el área de salud.

- Obtener información actualizada sobre cuestiones atinentes a educación, promoción y prevención de la salud

- $\quad$ Conocer asistencia básica en atención medica

Las metas del proyecto:

a. $\quad 500$ personas directamente.

b. $\quad 200$ alumnos becados vinculados a la comunidad.

c. 1 Informe Socio - Económico.

Las 4 etapas del proyecto fueron, etapa:

1. Socialización del Proyecto, etapa

2. Realización de un diagnostico Socio Económico

3. Charlas de Concienciación

4. Diagnostico y control antropométrico.

Las actividades correspondientes fueron ejecutadas en las fechas estipuladas en el cronograma de ejecución del proyecto.

\section{Calendario}

a. Inicio del Proyecto: 19/01/2012

b. Fin del Proyecto : 30/03/2012

\section{CONCLUSIONES}

En esta actividad se invirtieron 56 horas distribuidas en 38 actividades, con una participación de aproximadamente 1.310 personas entre alumnos, docentes de la UNC@y alumnos, profesores, directivos de la escuela 1ro de marzo, padres de familia y la comunidad del barrio primero de marzo.

De esta lógica de intervención se trabajo el componente de la transformación cultural de las personas al insertar en ellos las practicas de prevención y promoción de la salud, en el primer eslabón de la educación, educando a un niño el cual adoptara una nueva conducta, reforzada con 
los materiales didácticos diseñados para darle un seguimiento a las acciones planteadas con un cuadernillo de ejercicios que el niño debe completar con su profesor durante el periodo lectivo escolar, de esta forma los hábitos de prevención y fomento de la salud, mediante las practicas higiénicas y alimenticias contribuirán a fortalecer las acciones encaminadas para solucionar la presencia de parásitos y sus problemas colaterales.

La Extensión Universitaria de la UNC@, a través de la R.S.U. se propone involucrase en la realidad departamental y nacional para fomentar el aprendizaje continuo, mediante programas, proyectos y actividades que nos permitan la vinculación efectiva en las comunidades y mediante esto incrementar la gestión de las mismas en post del desarrollo sustentable.

Es sabido que la educación hace que haya conocimiento, este hace mejor la vida del ciudadano, este a su vez mejora la vida de su familia y la familia de la sociedad, es importante que la Universidad se vincula de manera coherente, con teorías que sean practicas a la hora de coadyuvar a la comunidad a un crecimiento y fortalecimiento integral y continuo de manera a cumplir con la labor de la Responsabilidad Social Universitaria.

\section{BIBLIOGRAFÍA}

González, O., Fontaneda I. y Camino M., (2010), La Responsabilidad Social en las Universidades Españolas 2010, http://books.google.com.py/books?id=QLlA_zxDf38C\&dq=editions:TOdU0Mcz6KQC\&hl=es\&s ource $=$ gbs_navlinks_s.

Cuesta, M y Sanchez, D. 2012 Responsabilidad Social Universitaria, netbiblo. España 2012 www.netbiblo.com

Comisión Europea (2001), Libro Verde, Dykinson 2006, Madrid, España.

Ramos, Nestor, (2012), La Responsabilidad Social Universitaria, Eae, editorial, España. 2012.

Gamboa, Jose Erik, (2012), Intervencion de Responsabilidad Social Universitaria, Editorial Academia Española, 2012. España. 


\section{ANEXOS}

Grafico 1 Cumplimiento de los objetivos del proyecto

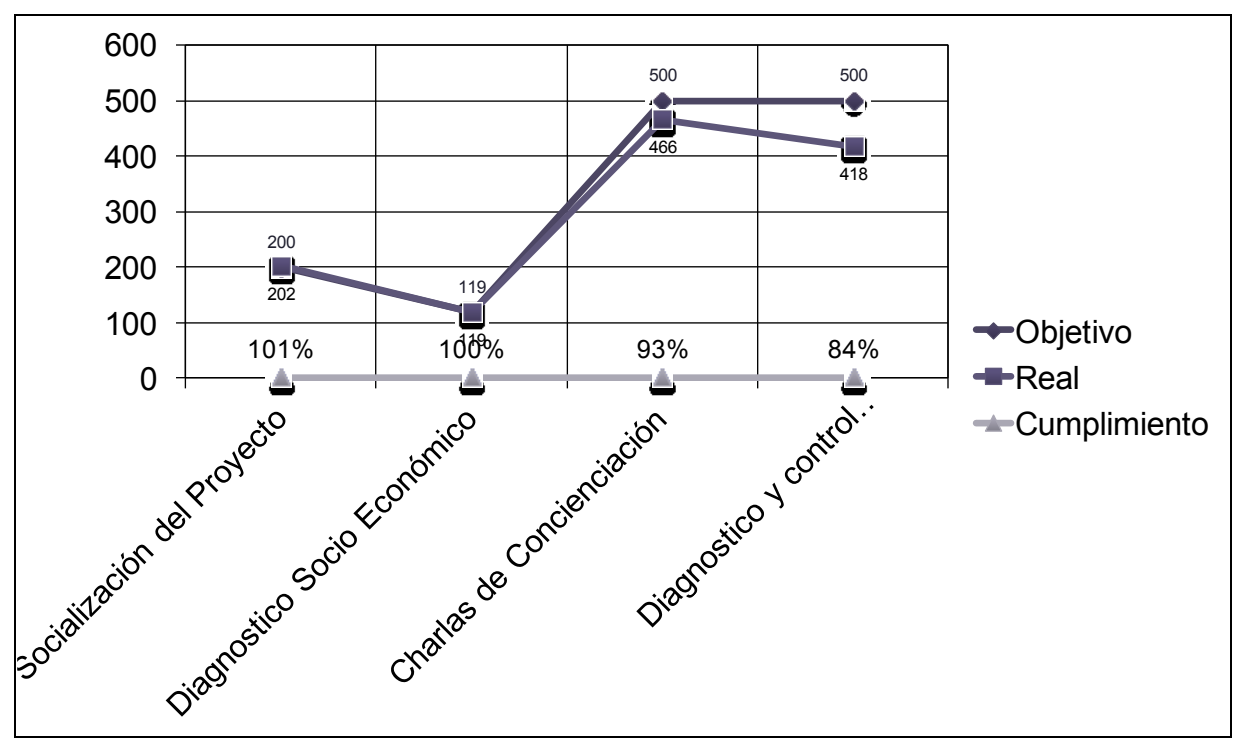

Grafico 2 Distribución de Medicamentos

Entrega de Medicamentos Turno Mañana

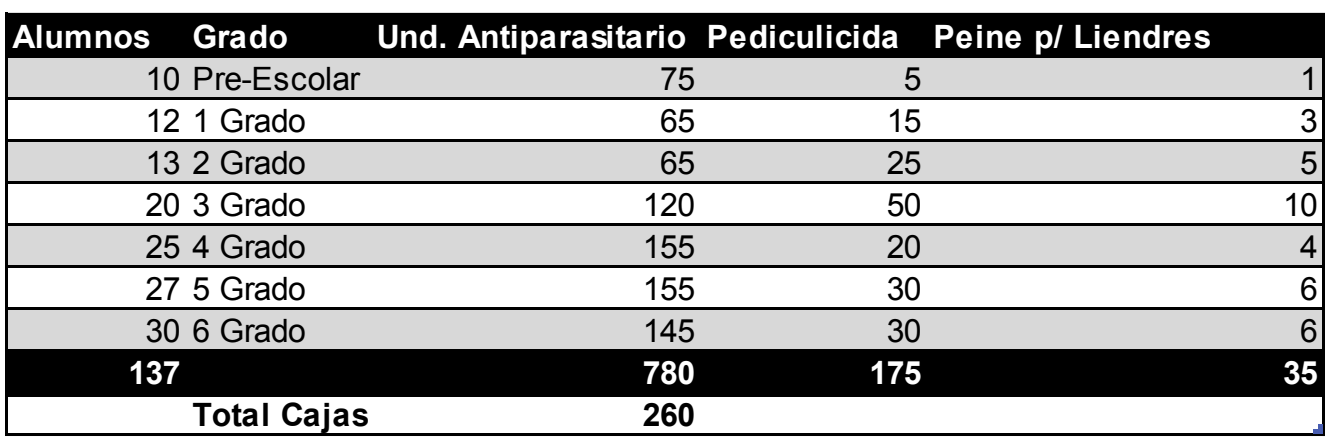

Entrega de Medicamentos Turno Tarde

\begin{tabular}{|crrrr|}
\hline Alumnos Grado & Und. Antiparasitario Pediculicida & Peine $\mathbf{p}$ / Liendres & \\
\hline 14 Jardin & 70 & 18 & 9 \\
\hline 14 Pre-Escolar & 70 & 6 & 3 \\
\hline 29 1 Grado & 145 & 30 & 15 \\
\hline 32 2 Grado & 160 & 4 & 7 \\
\hline 123 Grado & 60 & 12 & 12 \\
\hline 194 Grado & 95 & 26 & 13 \\
\hline 165 Grado & 80 & 8 & 5 \\
\hline 246 Grado & 135 & 10 & 5 \\
\hline $\mathbf{1 6 0}$ & $\mathbf{8 1 5}$ & $\mathbf{1 1 4}$ & $\mathbf{6 9}$ \\
\hline
\end{tabular}


Grafico 3 Participación de Alumnos
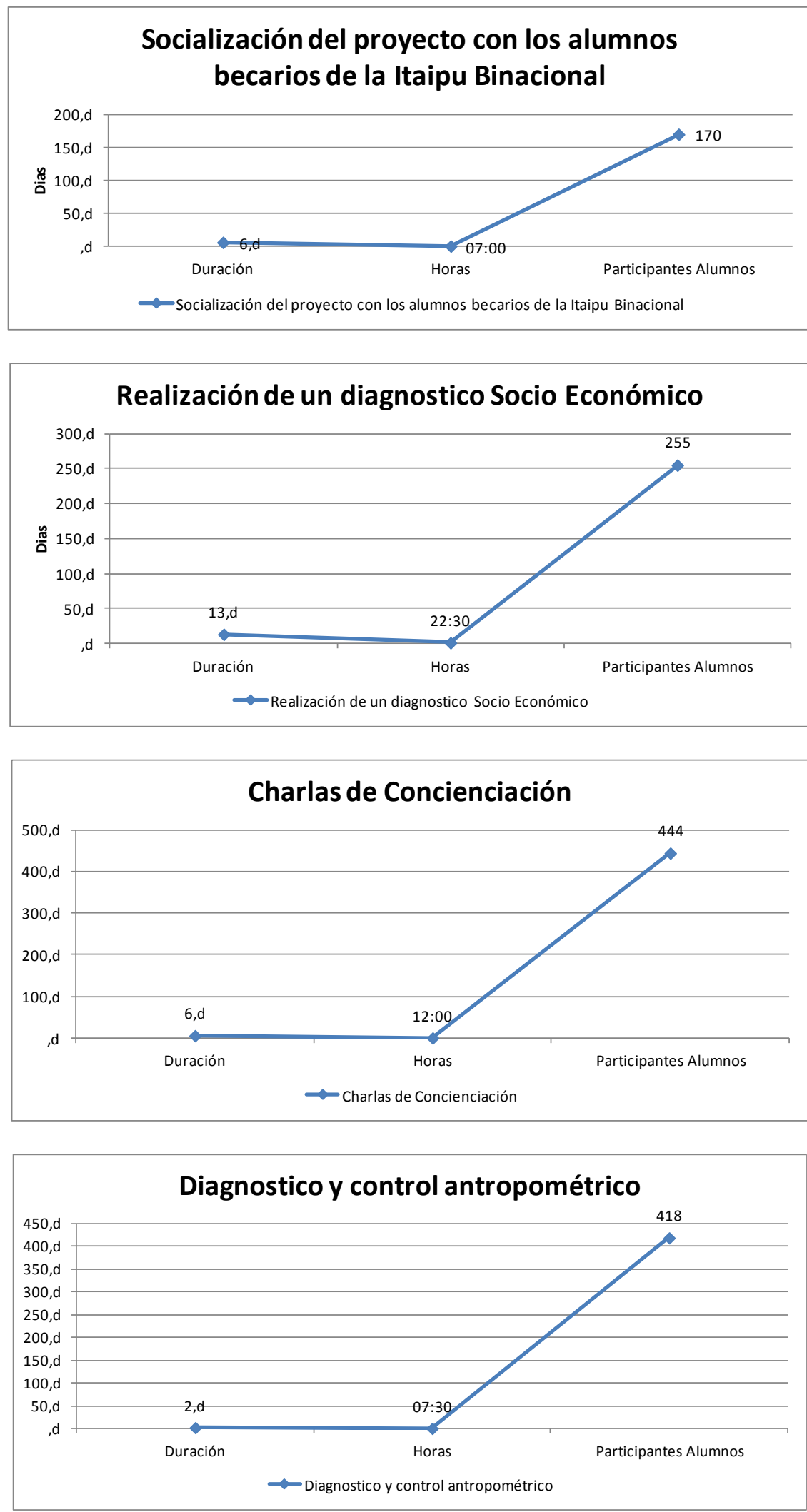
CRONOGRAMA DE ACTIVIDADES

PROYECTO: UN CAMBIO EN EL BARRIO 1RO DE MARZO

\begin{tabular}{|c|c|c|c|c|c|c|c|c|c|c|}
\hline Id & Nombre & Duración & Comienzo & Fin & $\begin{array}{l}\text { Hora } \\
\text { Inicio }\end{array}$ & $\begin{array}{c}\text { Hora } \\
\text { Fin }\end{array}$ & Horas & $\begin{array}{c}\text { Participantes } \\
\text { Docentes }\end{array}$ & $\begin{array}{l}\text { Participantes } \\
\text { Alumnos }\end{array}$ & Total \\
\hline & Socialización del proyecto con los alumnos becarios de la ltaipu Binacional & 1 & $19 / 01 / 2012$ & $19 / 01 / 2012$ & $16: 00$ & $17: 00$ & $01: 00$ & 3 & 37 & 40 \\
\hline & $\begin{array}{l}\text { Reunión de carácter técnico en la oficina de la Itaipu Binacional con los coordinadores } \\
\text { del proyecto }\end{array}$ & 1 & 23/01/2012 & $23 / 01 / 2012$ & 09:30 & $11: 30$ & 02:00 & 5 & 0 & \\
\hline & Socialización con docentes y personal académico de la FCE & 1 & $25 / 01 / 2012$ & $25 / 01 / 2012$ & 18:00 & 19:00 & $01: 00$ & 9 & 38 & 47 \\
\hline 5 & Socialización con los sub coordinadores del proyecto & 1 & $26 / 02 / 2012$ & $26 / 02 / 2012$ & $09: 30$ & $10: 30$ & $01: 00$ & 3 & 2 & 5 \\
\hline 6 & Socialización del Proyecto con personal de apoyo académico & 1 & $26 / 02 / 2012$ & $26 / 02 / 2012$ & $15: 00$ & $16: 00$ & $01: 00$ & 6 & 0 & 6 \\
\hline & Reunión técnica y aclaración de dudas con los alumnos becarios de la Itaipu & 1 & $27 / 01 / 2012$ & $27 / 01 / 2012$ & $18: 00$ & 19:00 & 01:00 & 6 & 93 & 99 \\
\hline & Elaboración del Instrumento & $1, \mathrm{~d}$ & $02 / 02 / 2012$ & $02 / 02 / 2012$ & $15: 30$ & $16: 30$ & $01: 00$ & 1 & 10 & 11 \\
\hline & Validación del Instrumento & $1, \mathrm{~d}$ & $06 / 02 / 2012$ & $06 / 02 / 2012$ & 16:00 & 17:00 & 01:00 & 2 & 4 & 6 \\
\hline & Capacitación a Encuestadores & $1, \mathrm{~d}$ & $07 / 02 / 2012$ & $07 / 02 / 2012$ & $08: 00$ & $09: 00$ & $01: 00$ & 2 & 12 & 14 \\
\hline & Distribución y elaboración de grupos de trabajo para la encuesta & $1, \mathrm{~d}$ & $09 / 02 / 2012$ & $09 / 02 / 2012$ & $17: 00$ & $18: 00$ & $01: 00$ & 1 & 42 & 43 \\
\hline & Aplicación de la encuesta F.C.E. & $\frac{1, \mathrm{~d}}{1, \mathrm{~s}}$ & $\frac{0 / 1 / 02 / 2012}{10}$ & $\frac{0 / 1 / 02 / 2012}{1 / 2}$ & $13: 00$ & $17: 00$ & $04: 00$ & $\frac{1}{6}$ & 125 & 131 \\
\hline 16 & Aplicación de la encuesta F.C.E. & $1, d$ & $12 / 02 / 2012$ & $12 / 02 / 2012$ & $10: 00$ & $12: 00$ & $02: 00$ & 6 & 26 & 32 \\
\hline & Construcción de una matriz en Excel & $1, \mathrm{~d}$ & $13 / 02 / 2012$ & $13 / 02 / 2012$ & $17: 00$ & $19: 30$ & $02: 30$ & 1 & 8 & 9 \\
\hline 18 & Procesamiento de los datos & $1, \mathrm{~d}$ & $15 / 02 / 2012$ & $15 / 02 / 2012$ & $17: 00$ & $18: 00$ & $01: 00$ & 1 & 8 & 9 \\
\hline & Preparación de los materiales & $1, \mathrm{~d}$ & $07 / 03 / 2012$ & $07 / 03 / 2012$ & $14: 00$ & $15: 00$ & $01: 00$ & 4 & 2 & 6 \\
\hline & Preparación de los alumnos & $\frac{1, d}{1, d}$ & $09 / 03 / 2012$ & $09 / 03 / 2012$ & $14: 00$ & $15: 00$ & $01: 00$ & 4 & 1 & 5 \\
\hline & Realización de las Charlas & $1, \mathrm{~d}$ & $13 / 03 / 2012$ & $13 / 03 / 2012$ & 07:00 & 11:00 & 04:00 & 4 & 129 & 133 \\
\hline & Realización de las Charlas & $1, \mathrm{~d}$ & $16 / 03 / 2012$ & $16 / 03 / 2012$ & $16: 00$ & $18: 00$ & $02: 00$ & 3 & 98 & 101 \\
\hline$\frac{-1}{28}$ & Distribución de Materiales Impresos casa por casa & $\frac{\mathrm{d}, \mathrm{u}}{1, \mathrm{~d}}$ & $24 / 03 / 2012$ & $24 / 03 / 2012$ & $13: 00$ & $16: 00$ & $03: 00$ & $\frac{5}{3}$ & 211 & 214 \\
\hline & Diagnostico y control antropométrico & $2, \mathrm{~d}$ & & & & & $07: 30$ & 34 & 326 & 360 \\
\hline & Realización del control antropométrico y Administración de la dosis de antiparasitario & $1, \mathrm{~d}$ & $29 / 03 / 2012$ & 29/03/2012 & $07: 30$ & 11:30 & 04:00 & 22 & 151 & 173 \\
\hline & Realización del control antropométrico y Administración de la dosis de antiparasitario & $1, \mathrm{~d}$ & $30 / 03 / 2012$ & $30 / 03 / 2012$ & 13:30 & 17:00 & 03:30 & 12 & 175 & 187 \\
\hline 36 & Cierre del proyecto & $5, \mathrm{~d}$ & & & & & & & & \\
\hline & Preparación de Rendición de Cuentas & $2, \mathrm{~d}$ & $09 / 04 / 2012$ & $20 / 04 / 2012$ & & & & & & \\
\hline & Elaboración del Informe Final & $\frac{2, \mathrm{u}}{1, \mathrm{~d}}$ & $09 / 04 / 2012$ & $20 / 04 / 2012$ & & & & & & \\
\hline
\end{tabular}

